UCRL-ID-120740

\title{
Spontaneous Origin of Topological Complexity in the Cerebral Cortex
}

\author{
George Chapline \\ University of California \\ Lawrence Livermore National Laboratory \\ Livermore, CA 94550
}

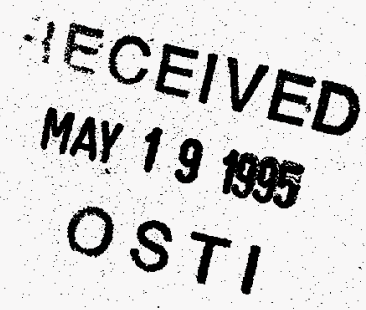

April 7, 1995

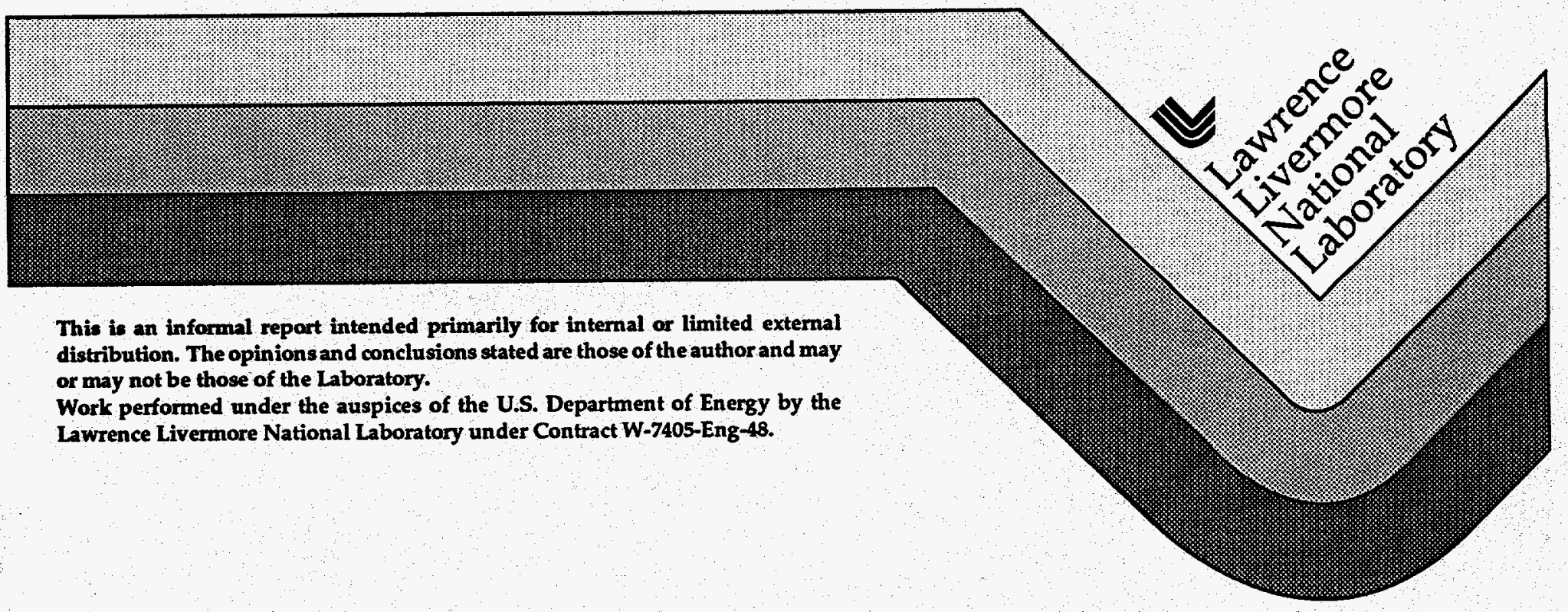




\section{DISCLAIMER}

This document was prepared as an.acccount of work sponsored by an agency of the United States Government. Neither the United States Government nor the University of California nor any of their employees, makes any warranty, express or implied, or assumes any legal liability or responsibility for the accuracy, completeness, or usefulness of any information, apparatus, product, or process disclosed, or represents that its use would not infringe privately own rights. Reference herein to any specific commercial products, process, or service by trade name, trademark, manufacturer, or otherwise, does not necessarily constitute or imply its endorsement, recommendation, or favoring by the United States Government or the University of California. The views and opinions of authors expressed herein do not necessarily state or reflect those of the United States Government or the University of California, and shall not be used for advertising or product endorsement purposes.

This report has been reproduced directly from the best available copy.

Available to DOE and DOE contractors from the Office of Scientific and Technical Information P.O. Box 62, Oak Ridge, TN 37831

Prices available from (615) 576-8401, FTS 626-8401

Available to the public from the National Technical Information Service

U.S. Department of Commerce 5285 Port Royal Rd. Springfield, VA 22161 


\section{DISCLAIMER}

Portions of this document may be illegible in electronic image products. Images are produced from the best available original document. 
Spontaneous Origin of Topological Complexity in the Cerebral Cortex

\author{
George Chapline
}

\begin{abstract}
Attention is drawn to the possibility of regarding the cerebral cortex as a physical system whose only excitations are topological. An attractive feature of such a hypothesis is that it is possible to understand how local dynamics could spontaneously give rise to a large scale organization of neurons and synapses that one might associate with sophisticated cognitive capabilities. It is suggested that the spontaneous appearance of topological disorder in the topological phases of 2-D and 4-D quantum gravity illustrates how the topological complexity of the human brain can develop. In particular the cooperative behavior of different neural circuits in the cerebral cortex may be closely related to the topology of certain 4-manifolds.
\end{abstract}


1. Introduction

Although the notion of intelligence has yet to acquire a precise definition, it is reasonable to surmise that the ability of the human brain to process and retain information in an intellectually sophisticated manner is intimately related to the topological complexity of the synaptic connections in the cerebral cortex. Just how the patterns of synaptic connections in the cerebral cortex are established is somewhat mysterious, particularly in view of the fact that the human genome doesn't contain nearly enough genetic information to specify how all the neurons in the cortex should be interconnected. It is also noteworthy that humans poses mental capabilities such as reading that could not have evolved by natural selection. In this report we wish to point out that the topological complexity of the connections in the cerebral cortex could have arisen spontaneously, in much the same way that topological disorder can arise in a two dimensional physical system from the appearance of Kosterlitz-Thouless vortices.

One of the central questions of neural science has been to what extent the human brain is like a computer. It has been argued [1] that the human brain is fundamentally different from a computer because computer programs have no intrinsic physical significance. However, one thing that computers and the human brain clearly have in common is that specified inputs should lead to predictable outputs. Therefore in some sense the human brain must incorporate the equivalent of a computer program, and apparently this equivalent of a computer program must have some physical interpretation. In the following we would like to draw attention to the possibility that the requirement that specified inputs should yield unique outputs can be taken to mean that only the boundary conditions for the system have physical significance. In other words, the only observable quantities for the system are purely topological [2]. The global behavior of such a system will be determined not only by the boundary conditions but also by the topology of the space in which the system lives. Thus the topology of an underlying manifold plays to some extent the same role as the program in an ordinary computer.

A physical system with purely topological excitations can be described either in terms of stochastic equations [3], or using an action which is a topological invariant [4]. The dynamics of these systems is unusual in that the only stationary states are ground states, and these ground states have a topological interpretation [3]. In the case of the human brain the physical system we wish to describe is the network of neurons and synaptic connections, and the underlying "manifold" in this case is a nonplanar graph whose edges correspond to synaptic connections. Over 
the past decade there has been great interest in the idea [5] that the behavior of the neurons and synaptic connections in the human brain can be understood by studying the behavior of an equivalent electric circuit, whose connectivity is the same as the nonplanar graph of synaptic connections. The behavior of this electric circuit will obviously depend on the topology of the electrical connections.

However, in order to make explicit contact with the topological field theories which have been recently used by mathematicians to study the topology of low dimensional manifolds it might be more useful [2] to regard the system of neurons and synaptic connections as a field theory defined on a 2-dimensional surface, whose topological genus is the same as that of the nonplanar graph of synaptic connections.

In this report we shall be concerned not so much with what particular physics model might be most useful for describing the behavior of the system of neurons and synaptic connections in the human brain, as how the nontrivial topology of the synaptic connections might arise. We wish to put forward the idea that the nontrivial topology of the synaptic connections arises spontaneously, with perhaps only the level of topological complexity being predetermined. In the following sections we will introduce three analytically solvable mathematical physics models which illustrate how a nontrivial topology for the synaptic connections might arise. The first two models pertain to how feature detectors within a single layer of the cerebral cortex can self-organize to produce vortex structures similar to the pinwheel-like singularities in the organization of orientation preference columns that have been observed in the primary visual cortex of monkeys [6]. We will argue that these pinwheel-like singularities are evidence that the orientation sensitive neurons within a single layer of the visual cortex are interconnected in a topologically nontrivial way. In section 4 a field theory model is introduced which provides a topological description for a foliation of feature detecting networks. This last model illustrates, at least in principle, how local dynamics can spontaneously give rise to a cooperative assembly of neural circuits, each of which is specialized to detect different features.

\section{Self-organization of Orientation Columns}

Pattern formation by self-organization may be a common phenomenon in brain development [7]. For example, numerical simulations based on Kohonen's self-organizing map algorithm have successfully reproduced qualitative features of the organization of orientation preference and ocular dominance columns within 
each hypercolumn of the visual cortex of the macaque monkey [8]. A particularly interesting result of these simulations is the occurrence of vortex-like singularities in the arrangement of orientation columns. The authors of ref. 8 interpreted the occurrence of these vortex singularities in terms of singularities that occur in dimension reducing maps. In the following we would like to offer an alternative topological interpretation for these singularities, based on a simple neural network model for self-organization of orientation preference columns.

Our basic network consists of $F$ feature detectors such that each feature detector is connected to three neighboring feature detectors. The assumption of three connections per neuron is made for convenience since models where the feature detectors are allowed to connect to larger numbers of neighbors lead to similar results. Also in this report we will concentrate on the case where each feature detector is characterized by an angle $\mathrm{v}$. For example, the orientation sensitive neurons within the primary visual cortex are characterized by a preferred orientation which denotes the stimulus orientation which gives the strongest response.

We wish to develop a theory for how feature detectors characterized by an angle are organized within a single layer of the cerebral cortex. As our starting point we consider maps that assign to each preferred orientation $\mathrm{v}$ a location $\underline{r}$ within our network of F feature detectors. Following Kohonen [7] we will assume that brain development can be modeled by assuming that the maps of interest are "selforganizing". That is initially each feature detector is assigned a random orientation $w(r, 0)$, and each orientation $v$ in the environment is mapped to that feature detector $\mathrm{r}$ whose orientation $\mathrm{w}(\mathrm{r}, 0)$ is closest to $\mathrm{v}$. Thereafter the orientation of the feature detector located at $r$ evolves according to a rule of the form

$$
w(r, t+1)=w(r, t)+h(r-s)[v-w(r, t)]
$$

where $h(x)$ is typically assumed to be a Gaussian function peaked at $x=0$. In our case the function $\mathrm{h}(\mathrm{r}-\mathrm{s})$ will be replaced by the rule that each feature detector is connected only to its three nearest neighbors. The location $s$ in (1) corresponds to the feature detector whose orientation $\mathrm{w}(\mathrm{s})$ is closest to $\mathrm{v}$. Thus the developmental process is modeled as a Markov process whose states are the sets $\{\mathrm{w}(\mathrm{r})\}$ of possible orientations, and where the transition probabilities are determined by probabilities of occurrence in the environment of various orientations $v$. In order to construct an analytical 
model of this developmental process it will be useful to introduce an energy functional $E[w]$ that satisfies

$$
<d w P(v)>=-\operatorname{grad}_{w} E,
$$

where $P(v)$ is the probability distribution for the orientations of environmental stimuli. Neglecting certain mathematical subtleties, the required energy functional is [9]

$$
E[w]=\frac{1}{2} \sum_{<r, s>} \sum_{v \in R(r)} P(v)|v-w(r, t)|^{2}
$$

where the sum over $\langle\mathrm{r}, \mathrm{s}\rangle$ runs over nearest neighbor connections and $\mathrm{R}(\mathrm{r})$ is the receptive field of the feature detector located at $r$; i.e. the union of all environmental stimuli that are closer to $w(r, t)$ than any other $w(s, t)$, where $s \neq r$.

Given an energy functional that satisfies (2) there are standard techniques that one can use to investigate the stochastic evolution of the organization of our feature detecting network. However in the following our only interest in how the selforganization of our feature detectors evolves with time will be to note that under the influence of the random variable $v(t)$ which describes the effect of the environment the system relaxes to an asymptotic state characterized by a stationary probability distribution for various final configurations $\{w(r)\}$. The statistical properties of our network of feature detectors in this stationary state can be derived from a "partition function", which is a sum over all possible stationary state configurations weighted with the Boltzmann factor exp (-E[w]). If we assume that the stochastic evolution of our network is governed by an energy functional of the form (3) then this partition function has the form:

$$
Z=\sum_{L} \kappa^{F} \prod_{i=l}^{F} \int_{o}^{2 \pi} d w\left(r_{i}\right) e^{-\frac{K}{2} \sum_{i, j, j} \operatorname{lw}\left(r_{i}\right)-\left.w\left(r_{j}\right)\right|^{2}}
$$

where $\kappa$ and $\mathrm{K}$ are constants, the sum over $\mathrm{L}$ means a sum over triangular lattices, and the indices $i$ and $j$ refer to orientation sensitive neurons located at the centers of the triangles in this lattice (note that $\mathrm{F}$ is the number of faces of the lattice $\mathrm{L}$ ). For large numbers of faces $\mathrm{F}$ the triangular lattices $\mathrm{L}$ can be thought of as triangulations of 2-dimensional surfaces, and in the limit $F \rightarrow \infty$ the sum over triangular lattices in 
(4) becomes a sum over smooth 2-dimensional surfaces. In this limit the partition function (4) becomes

$$
Z=\int D w(\sigma) \exp (-S)
$$

where $\sigma$ is the coordinate of a point on the smooth surface and the continuum action $\mathrm{S}$ is given by

$$
S=\frac{K}{2} \int d^{2} \sigma \partial_{\alpha} w \partial_{\alpha} w+\lambda
$$

The constant $\lambda$ in (6) replaces the constant $\kappa$ and plays the role of energy per neuron. Partition functions using classical actions similar to (6) were originally introduced as quantum theories of matter coupled to 2-D gravity [10]. In particular, the quantum theory defined by (5) describes the coupling of 2-D quantum gravity to a single scalar field, and has been intensively studied by mathematical physicists [11]. If one assumes that this scalar field represents a periodic variable, then it turns out that there is a phase of the theory where the dynamics is essentially the dynamics of 2-D quantum gravity.

Another interpretation [11] of the partition function (5) is that it represents a relativistic string moving on a 2-dimensional surface - in mathematical terms this means holomorphic mappings from an arbitrary 2-dimensional manifold onto a fixed 2-dimensional manifold. It is worth noting that this result is consistent with the theorem [12] that for maps of 2-dimensional surfaces onto 2-dimensional surfaces the stationary state of Kohonen's algorithm is a holomorphic (or antiholomorphic) map. Thus we arrive at the general result that the coordinate $\mathrm{w}$ is a function of $z=x+i y$. or $\bar{z}=x-i y$. Of particular interest to us here are solutions where $\mathrm{w}(\mathrm{z})$ has the form where the $\mathrm{m}_{\mathrm{i}}$ are integers. In general one must have

$$
w(z)=\sum_{i} m_{i} \operatorname{Im} \ln \left(z-Z_{i}\right)
$$

$\sum \mathrm{m}_{\mathrm{i}}=0$. In fact these solutions are just the vortex configurations of the 2dimensional XY model discovered by Kosterlitz and Thouless [13]. Substituting the configuration (7) into the action (6), the path integral (5) assumes a form identical to 
the partition function for a 2-dimensional Coulomb gas, with $1 / \pi \mathrm{K}$ playing the role of temperature:

$$
Z_{v}=\sum_{m_{i}, Z_{i}} \exp \left[-\pi K\left\{\sum_{i \neq j} m_{i} m_{j} \ln \frac{l}{\left|Z_{i}-Z_{j}\right|}\right\}\right]
$$

As was first pointed out by Kosterlitz and Thouless [13] a 2-dimensional Coulomb gas has a phase transition which implies that at low temperatures the vortexantivortex pairs in the system (8) are bound together, while at high temperatures they are dissociated. Although the exact dependence of the string theory partition function (5) on temperature is not quite the same as for the $X Y$ model, it can be shown [14] that the basic picture of a Kosterlitz-Thouless (KT) phase transition holds in string theory. Applied to our network of orientation sensitive neurons the existence of a KT phase transition means that if the constant $\mathrm{K}$ is less than a certain critical value then vortex-like configurations should be a prominent feature of the organization of orientation preference columns in the primary visual cortex of all primates.

\section{2-D Quantum Gravity Model}

As mentioned in the last section our theory of self-organized orientation columns can also be interpreted as a theory of 2-dimensional quantum gravity coupled to a scalar field representing orientation preference. Inspection of the action (6) reveals that when the coupling constant $K$ is very small one can neglect kinetic variations in the scalar field. This suggests an alternative approach to understanding the appearance of vortex configurations in the pattern of orientation columns. To begin with we assume that the scalar field associated with orientation preference is frozen into a final configuration and no longer need be treated as a fluctuating field. On the other hand the "quantum gravity" degrees of freedom are still active. This means that when $F$ is large and the coupling $K$ is less than the critical value $K_{c}=2 / \pi$ our theory of self organized feature detectors effectively becomes a theory of random surfaces, where points of the random surface are labeled with a fixed orientation preferences. This interpretation of the "weak coupling" phase for our network of feature detectors leads us to an alternative topological interpretation for the occurrence of the vortex configurations. 
For a fixed number $\mathrm{F}$ of feature detectors the activation of "quantum gravity" degrees of freedom essentially means that the 2-dimensional surface approximated by a triangular lattice that is dual to the network of feature detectors will change with time. This does not mean that the local connections between feature detectors will change, but the global way this network of feature detectors folds back on itself will be allowed to vary (the network can be thought of as a discretization of a 2dimensional surface with varying topology). In fact when both $\mathrm{F}$ is large and the constant $\kappa$ approaches a certain critical value, the topological genus can become very large [15]. It is not hard to show that the projection of a 2-dimensional surface with a large topological genus onto the smooth two dimensional surface that one might naively associate with a single layer of the visual cortex will necessarily lead to singularities that look like the KT vortices (7). In particular one can make use of the Riemann- Hurwitz mapping theorem [16], which relates the topological indices of a holomorphic mapping between two 2-dimensional manifolds to the topological genus of each surface. The topological indices that appear are the ramification indices $n_{i}$ which describe the behavior of the mapping near singular points and the winding number $\mathrm{n}$ which describes the number of times the mapped surface is covered by the mapping. In our case we are interested in mapping the surface associated with our network of feature detectors to the disc-like region constituting a single layer of a hypercolumn within the visual cortex. In the case where one is mapping a surface of genus $g$ to a disc the Riemann-Hurwitz relation reduces to the simple formula

$$
\sum_{i}\left(n_{i}-1\right)-n=2 g-2
$$

where the sum runs over all singular points. If $g$ is positive and large then it follows that there must be some $n_{i}>0$. In addition, the sum $\sum n_{i}$ must be large, so that if there are only a few singular points per hypercolumn the $n_{i}$ themselves must be large. Indeed it is reasonable to assume that the $n_{\mathbf{i}}$ have magnitudes on the order of the number of orientations that the human brain can distinguish; i.e. a few thousand. Since near a singular point the mapping can be approximated as $\mathrm{z}^{\mathrm{n}_{\mathrm{i}}}$ [16], these singular points begin to look a lot like the pinwheel-like patterns of orientation preference columns observed in the visual cortex of monkeys [6]. Therefore we reach the conclusion that the unusual pattern of orientation preference columns observed in the visual cortex of monkeys may be a signal that in 
primates the orientation sensitive neurons within a single hypercolumn of the visual cortex are connected together in a topologically nontrivial way, and furthermore this topologically nontrivial pattern of connections can appear spontaneously as a result of self-organization. A topologically nontrivial pattern of synaptic connections is, in fact, characteristic of the recurrent networks that are thought to play a significant role in human cognitive capabilities [17].

\section{4-D Quantum Gravity Model}

Although the cerebral cortex is layered the neurons in different layers interact with each other. More generally, one of the most characteristic features of mental processes is that they involve cooperation of neural circuits at different locations within the cerebral cortex. These different neural circuits typically are specialized for recognition of different aspects of an object or sound. In this section we will show how the theory of self-organization of orientation preference columns developed in section 2 can be extended to describe a foliation of feature detecting networks, where each network in the foliation contains slightly different feature detectors. We begin by slightly altering the lattice version of the continuum theory of section 2 .

In order to recognize the fact that the orientation preferences $w[r]$ are periodic variables one can replace the exponential link factors in the partition function (4) with a Villain link factor $\sum_{m=-\infty}^{\infty} \exp \left[-\frac{K}{2}(w+2 \pi m)^{2}\right]$, where the sum over $m$ insures that the link factor is periodic under $w->w+2 \pi$. Now the partition function (4) has the form

$$
Z=\sum_{L} \kappa^{F} \prod_{i=l}^{F} \int_{0}^{2 \pi} d w\left(r_{i}\right) \prod_{<i j>m_{i_{i j}}} e^{-\frac{K}{2}\left[w\left(r_{i}\right)-w\left(r_{j}\right)+2 \pi m_{i j}\right]^{2}}
$$

where as before the sum over $L$ runs over triangular lattices and the feature detecting neurons are situated on the faces of this lattice. In this lattice model a vortex is associated with a particular vertex of a triangular lattice and has a topological charge

$$
M=\sum_{\text {loop }} m_{i j}
$$

where the sum runs over the links of the neural network surrounding the particular vertex of the triangular lattice. This discrete form of the string theory 
partition function is instructive because it shows that the topological charges of the $\mathrm{KT}$ vortices are formally identical with quantized magnet fluxes. Indeed if we were to introduce real magnetic fields into the lattice theory (10) we would obtain an antiferromagnetic version of the 2-dimensional $X Y$ model, that again contains topological excitations [18]. Such a model has much in common with models of spinglasses, which it may be recalled were one of the inspirations for Hopfield's neural network [5].

One may now make use of a trick previously introduced by the author [19] for generalizing a 2-dimensional antiferromagnetic $X Y$-like model to 3-dimensions. Namely, one replaces the effective magnet fields in (10) by a nonabelian magnetic field with many "colors". The previous holomorphic mapping condition now becomes the condition

$$
\left(D_{x}-i D_{y}\right) W=o
$$

where $D_{\alpha}=-i \partial_{\alpha}+\left[A_{\alpha}, A_{\alpha}\right.$ is the nonabelian gauge potential, and $\mathrm{W}$ is an multicomponent field that we wish to use to describe the feature preferences in a foliation consisting of $\mathrm{N}$ layers of feature detectors. The choice of magnetic field strengths is somewhat arbitrary; however, one elegant way to maintain the topological character of the theory is to replace the string action (6) with the topological action [20]:

$$
S_{\text {top }}=\int d^{2} \sigma \operatorname{Tr} \varepsilon^{\alpha \beta}\left\{\frac{1}{4} F_{\alpha \beta}\left[W^{+}, W\right]-D_{\alpha} W^{+} D_{\beta} W\right\}
$$

where $B=F_{12}$ and $W$ are assumed to belong to the adjoint representation of the Lie group SU(N). The classical equations of motion corresponding to the action (13) can be solved exactly [21]. Moreover in the limit $\mathrm{N} \rightarrow \infty$ the solution to these equations describes the geometry of a certain kind of 4-dimensional manifold [19]. Remarkably the geometry and topology of this manifold can be expressed in terms of the magnetic potential generated by magnetic monopole-like topological excitations [22]. Indeed in the limit $\mathrm{N} \rightarrow \infty$ the effective magnetic field will be given by

$$
B(X)=\sum_{k} \operatorname{grad}_{X_{k}}\left\{\frac{l}{\left|X-X_{k}\right|}\right\}
$$


where the sum runs over the positions of the monopoles and the third coordinate specifies the layer of the foliation where the monopole is located. It can be shown that the magnetic monopole-like objects in (14) endow the 4-dimensional manifold with certain nontrivial topological characteristics [22], and the quantum theory corresponding to the $\mathrm{N} \rightarrow \infty$ limit of the action (13) can be interpreted as a model for the topological phase of 4-dimensional quantum gravity [23].

In the present context the magnetic field $B$ is merely a formal device for relating the feature detectors and synaptic connection strengths in different layers of the foliation of feature detecting networks. According to this model the nature of the feature detectors varies smoothly from layer to layer and is self-organized according to (12) within each layer. The self-organization principle (12) is a kind of twisted version of Kohonen self-organization. As in the 2-dimensional case topological complexity appears spontaneously because under certain conditions the monopole gas corresponding to (14) consists of dissociated monopoleantimonopole pairs. This implies that the feature detectors in our foliation of feature detecting networks are interconnected in a topologically nontrivial and subtle way. Furthermore it is interesting to note that because the topology of our foliation of networks is nontrivial in a 4-dimensional sense, "time" must play an essential role in establishing the cooperative behavior of this system. Actually the 4th dimension for the 4-manifolds of interest is not ordinary time ( see ref. 22), but instead is a periodic variable that is used to tie together the boundary conditions for the various layers in the foliation. The natural appearance of a periodic time variable in a topological description of a foliation of networks of feature detecting neurons is certainly intriguing in view of the suggestion [24] that visual awareness and other aspects of consciousness are the result of the rhythmic and synchronized firing of neurons in the different cortical areas concerned with the recognition of a particular object. Indeed one is very tempted to identify the periodic time coordinate of the 4-manifold associated with the effective magnetic field (14) with the 40-Hertz rhythm in the brain that is widely believed to be involved with consciousness.

\section{Conclusion}

One of the central mysteries of neural science is how the neurons and synapses in the cerebral cortex become organized to perform sophisticated cognitive functions. This mystery is deepened by the fact that the human genome fails by many orders of magnitude to contain enough information to specify how the immense number of neurons in the human brain should be interconnected. 
Evidently some principle of self organization must be at work. Kohonen's selforganizing maps have a certain physiological plausibility, but the question of how Kohonen's maps lead to sophisticated mental capabilities has remained unanswered. In this note we have pointed out that simple physical models that are self organizing in a way closely related to Kohonen's maps provide a natural explanation for how self-organization can spontaneously lead to topological structures that are very nontrivial. In particular, we have argued that a KosterlitzThouless transition in our models of self organizing feature detectors leads to recurrent networks with a topologically nontrivial pattern of connections. According to this view the detailed pattern of synaptic connections is not predetermined. However it is possible that the general level of topological complexity is genetically predetermined as a result of genetic specification of those physiological characteristics that affect pertinent self-organization parameters such as the biological equivalents of the parameters $\kappa$ and $K$ that occur in our idealized 2D model (5) .

The idea that cognitive processes are associated with topological excitations of the cerebral cortex is in accord with the well known fact that mental activities typically involve a number of different regions of the cortex. In addition, we have found that the cooperative behavior of different neural circuits in the brain may be rooted in the spontaneous appearance of structures that are topologically nontrivial in a 4-dimensional sense. The 4th dimension in this interpretation is an internal periodic time variable that serves the purpose of tying together the boundary conditions for the different neural circuits so that the different circuits are representing the same object. Thus the topological structure of certain 4-manifolds may provide a mathematical basis for understanding consciousness.

\section{References}

1. Searle, J. R. (1992) The Rediscovery of the Mind. ( MIT Press, Cambridge, Mass.).

2. The idea that the cerebral cortex might be described as a system with only topological excitations was first put forward by the author in a talk given at the Institute for Advanced Study, May 20, 1988. 
3. Witten, E. (1988) Comm. Math. Phys. 17 353-386.

4. Baulieu, L. and Singer, I. M. (1988) Nucl. Phys. Proc. Sup. 15B 12.

5. Hopfield, J. J. (1984) Proc. Natl. Acad. Sci. U.S.A. 81, 3088-3092.

6. Bonhoeffer, T. and Grinvald, A. (1991) Nature $\underline{353}$ 429-431.

7. Kohonen, T. (1987) Self-Organization and Associative Memory ( Springer Verlag, New York).

8. Obermayer, K., Ritter, H. and Schulten, K. (1990) Proc. Natl. Acad. Sci. U.S.A. 87, 8345-8349.

9. Ritter, H. and Schulten, K. (1988) IEEE International Conference on Neural Networks vol.I, 109-116.

10. Polyakov, A. M. (1981) Phys. Lett. 103B, 207.

11. Klebanov, I. R. (1992) "String Theory In Two Dimensions." Proceedings of the Spring School on String Theory and Quantum Gravity, Trieste, April,1991." ( World Scientific).

12. Ritter, H. and Schulten, K. (1986) Biol. Cybern. 54, 99-106.

13. Kosterlitz, J. M. and Thouless, D. J. (1973) J. Phys.C6, 1181.

14. Chapline, G. and Klinkhamer, F. (1989) Mod. Phys. Lett. A4, 1063-1067.

15. Gross, D. J. and Migdal, A. (1990) Phys. Rev. Lett. 64 , 717.

16. Farkas, Hershel M. and Kra, Irwin (1992) Riemann Surfaces ( Springer Verlag, New York).

17. Hinton, G. H. and Shallice, T. (1991) Psychological Review 98, 74-95. 
18. Einhorn, M. B. and Savit, R. (1978) Phys. Rev. D17 2583-2594.

19. Chapline, G. and Yamagishi, K. (1991) Phys. Rev. Lett. 66, 3064-3066.

20. Chapline, G. and Grossman, B. (1989) Phys. Lett. B223, 336-341.

21. Grossman, B. (1991) Phys. Rev. Lett. 65 , 3064-3066.

22. Gibbons, G. W. and Hawking, S. W. (1978) Phys. Lett. B78, 430-432.

23. Chapline, G. (1992) Mod. Phys. Lett. A7, 1959-1965.

24. Crick, F. and Koch, C. (1990) Seminars in the Neurosciences 2, 263-275. 